\title{
Photoluminescence investigation of GaN films grown by metalorganic chemical vapor deposition on (100) GaAs
}

\author{
C. H. Hong, D. Pavlidis, S. W. Brown, a) and S. C. Rand ${ }^{\text {a) }}$ \\ Solid State Electronics Laboratory, Department of Electronic Engineering and Computational Science, \\ The University of Michigan, Ann Arbor, Michigan 48109
}

(Received 8 August 1994; accepted for publication 3 November 1994)

\begin{abstract}
GaN films were grown on (100) GaAs substrates by metalorganic chemical vapor deposition and were found to be of (200) cubic or (111) cubic/(0002) hexagonal phase. Their photoluminescence characteristics remained invariant with material phase. We report assignment of band-edge photoluminescence near $3: 36 \mathrm{eV}$ and $3.15-3.31 \mathrm{eV}$ in apparently cubic GaN to intrinsic/bound excitons and phonon-assisted, donor-acceptor pair recombination respectively, on the basis of observed temperature and intensity dependences. A free exciton energy of $3.375 \mathrm{eV}$ is deduced at 6.5 K. ㅇ 1995 American Institute of Physics.
\end{abstract}

\section{INTRODUCTION}

GaN is one of a family of wide band gap nitride III-V semiconductors being actively investigated for applications in the area of opto-electronics. Nitrides like AlN and GaN exhibit direct gap emission and their ternary alloys are good prospects for ultraviolet light emitting diodes and lasers, electro-optic, piezo-electric, and acousto-optic modulators and for negative electron affinity devices. In the past, numerous investigations have concentrated on samples with wurtzitic crystal structure, but more recently samples with the zincblende structure have been prepared, principally by molecular beam epitaxy (MBE) and metalorganic chemical vapor deposition (MOCVD). Interest in cubic phase material has been motivated by a desire to explore its predicted superiority for certain device applications and to integrate $\mathrm{GaN}$ devices on readily available, high quality $\mathrm{III}-\mathrm{V}$ semiconductor substrates which minimize lattice mismatch with the nitride crystal structure. With these considerations in mind, we have studied the growth of $\mathrm{GaN}$ and reported the presence of cubic (c-GaN) epitaxial films on both (100) (Ref. 1) and (111) (Ref. 2) GaAs. This paper reports the optical properties of GaN epitaxial films grown on (100) GaAs substrates at temperatures below $550^{\circ} \mathrm{C}$, and our detailed studies of their optical properties in order to establish the origin of the main emission lines.

The hexagonal phase of GaN grows well on sapphire, and both its structure and optical properties have been studied extensively in the past. ${ }^{3-7}$ However, the insulating character of sapphire substrates has hampered progress toward large scale integration. Growth of cubic phase GaN films on silicon, ${ }^{8}$ silicon carbide, ${ }^{9}$ magnesium oxide, ${ }^{10}$ and gallium arsenide has been explored, with experiments on GaAs substrates having been reported only recently using MBE and MOCVD techniques. ${ }^{11-13}$ While structural characteristics of the material were carefully addressed in these earlier investigations, only limited aspects of luminescence properties of $c$-GaN were reported. Strite ${ }^{14}$ observed several cathodoluminescence emission peaks from $c-\mathrm{GaN}$ on GaAs in the vicin-

a) Division of Applied Physics, 1049 Randall Laboratory, The University of Michigan, Ann Arbor, MI 48109-1120. ity of $3.2 \mathrm{eV}$, as well as a very intense, broad luminescence band at midgap ascribed to defects. It was argued, chiefly by analogy with work on hexagonal $\mathrm{GaN}^{6,7}$ that several of the emission lines in their spectra were due to electron-bound hole and donor-acceptor (DA) pair recombination emission. Okumura $^{15}$ also performed cathodoluminescence experiments, and observed a broad luminescence band centered at $325 \mathrm{~nm}$, but there do not appear to be any prior reports of the intrinsic photoluminescence spectrum. Here we report temperature and excitation intensity dependences of wellresolved features of the photoluminescence spectrum near the band edge of (200)cubic or (111)cubic/(0002)hexagonal phase GaN which permit assignments of peaks to free exciton emission, phonon-assisted exciton recombination, and donor-acceptor (DA) pair processes.

\section{GaN GROWTH AND CHARACTERIZATION}

GaN films were grown on (100) semi-insulating GaAs substrates in a low pressure (60 Torr) MOCVD system containing using hydrogen (H2)carrier gas. Substrates were chemically cleaned in a solution of $\mathrm{H}_{2} \mathrm{SO}_{4}: \mathrm{H}_{2} \mathrm{O}_{2}: \mathrm{H}_{2} \mathrm{O}$ $=5: 1: 1$, mounted on a graphite susceptor and loaded into a horizontal quartz reactor. Halogen lamps were used to heat the substrate and temperature was monitored continuously with a thermocouple. The substrates were first annealed in an atmosphere of $\mathrm{H}_{2}$ and $\mathrm{AsH}_{3}$ at $650^{\circ} \mathrm{C}$ for $5 \mathrm{~min}$ to remove surface oxide. This step was followed by surface nitridation in which substrates were exposed to $1 \mathrm{sl} / \mathrm{min}$ of ammonia $\left(\mathrm{NH}_{3}\right)$ for $10 \mathrm{~min}$ at a temperature of $600^{\circ} \mathrm{C}$ and then lowered to the growth temperature of $530^{\circ} \mathrm{C}$. Trimethylgallium (TMG) and $\mathrm{NH}_{3}$ were supplied at a fixed V/III ratio of 3000 . The TMG flow rate was maintained at $8.9 \mu \mathrm{mole} / \mathrm{min}$ for all experiments reported here. Typical growth rates were on the order of $0.6 \mu \mathrm{m} / \mathrm{h}$, and total thicknesses of GaN films grown in this way were typically $0.35 \mu \mathrm{m}$. Analysis of $\mathrm{x}$-ray diffraction spectra and transmission electron microscopy measurements shows a transformation from (111) cubic or (0002) hexagonal to (200) cubic phase $\mathrm{GaN}$ as the growth temperature is raised from 530 to $600{ }^{\circ} \mathrm{C}$. Details of the x-ray characterization are presented elsewhere. ${ }^{16}$ The hexagonal phase is found to dominate as the temperature is further increased 


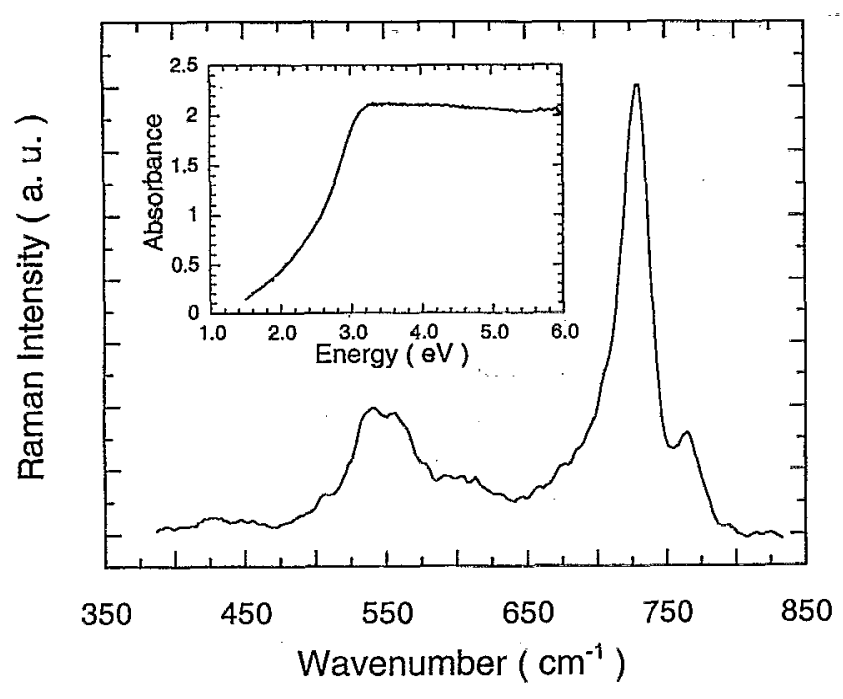

FIG. 1. The room temperature Raman spectrum of $\mathrm{GaN}\left(l_{\mathrm{ex}}=514.5 \mathrm{~nm}\right)$. Inset: The absorption spectrum of a free-standing GaN film of $2 \mu \mathrm{m}$ thickness.

above $650^{\circ} \mathrm{C}$ while the cubic phase dominated in the range of $570-650^{\circ} \mathrm{C}$. Post-growth annealing was performed for 20 $\min$ in an ammonia atmosphere at $670^{\circ} \mathrm{C}$.

For photoluminescence observations, a frequencydoubled picosecond DCM dye laser was used to provide quasi-continuous excitation at $325 \mathrm{~nm}$. Doubling was performed outside the dye laser cavity using an angle-tuned $\mathrm{LiIO}_{3}$ crystal at room temperature. Average powers on the order of $1 \mathrm{~mW}$ were easily obtained at a cavity-dumped, pulse repetition rate of $38 \mathrm{MHz}$ and light from this source was directed to samples mounted in an open-cycle liquid helium cryostat which permitted temperature control in the range $6-295 \mathrm{~K}$. Luminescence from the sample was then collected in a back-scattered geometry and focused onto the entrance slits of a $1 \mathrm{~m}$ spectrometer (ISA THR-1000). Photoluminescence (PL) signals were detected with a C31034A-02 GaAs photomultiplier in a photon-counting mode, and in this manner spectra were recorded as functions of both temperature and excitation intensity. All photoluminescence spectra presented in this paper correspond to films grown at $530^{\circ} \mathrm{C}$ which were of (111) cubic or (0002) hexagonal phase. Characterization of (200) cubic samples grown at higher temperatures revealed essentially the same photoluminescence characteristics with no shift in their position and thus no obvious difference of band gap. The only remarkable difference was the intensity of the spectra which increased for samples grown at higher temperatures. Although not possible to fully confirm at this stage, the results obtained in this work seem to correspond to cubic GaN films. Several samples were also etched using $10 \mathrm{~mL} \mathrm{NH} \mathrm{NH}_{4} \mathrm{OH}$ $500 \mathrm{ml}$ of $30 \% \mathrm{H}_{2} \mathrm{O}_{2}$ to yield free standing $\mathrm{GaN}$ films on which absorption measurements were made.

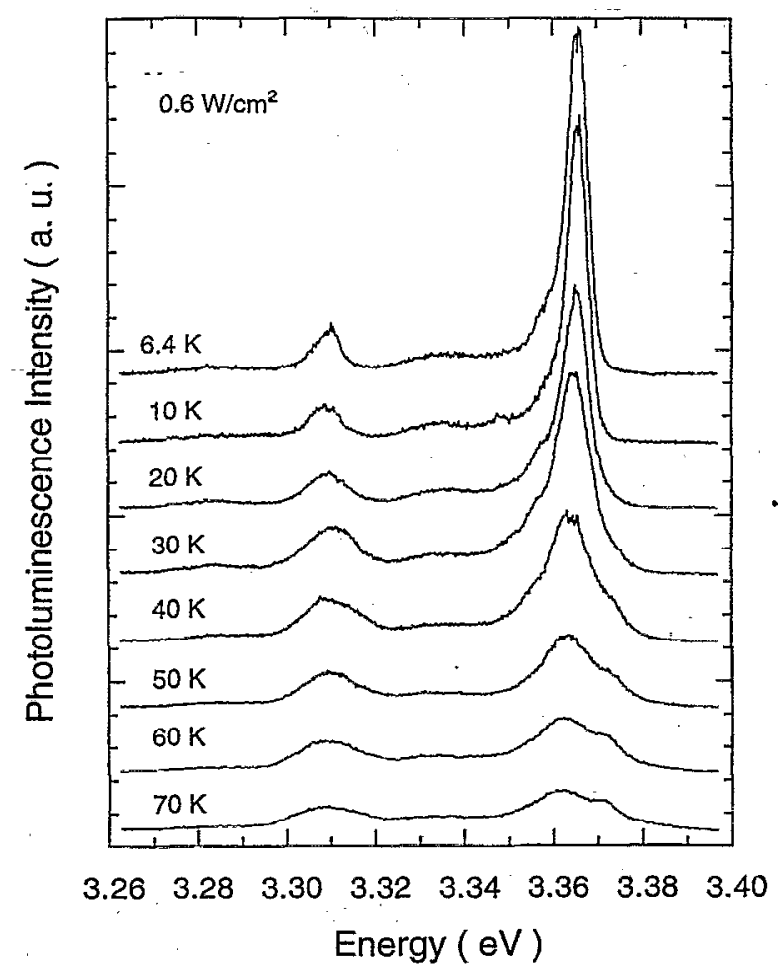

FIG. 2. High resolution photoluminescence spectra of near band-edge emission in GaN $\left(l_{\mathrm{ex}}=325 \mathrm{~nm}\right)$ vs temperature.

\section{EXPERIMENTAL RESULTS AND ANALYSIS OF PHOTOLUMINESCENCE CHARACTERISTICS}

In Fig. 1, the Raman spectrum of a typical GaN film recorded using $488 \mathrm{~nm}$ excitation light is shown, together with the absorption spectrum (inset). The Raman spectrum showed strong LO and TO components at 91.4 and 68.9 $\mathrm{meV}$, respectively, as expected. ${ }^{17,18}$ The GaAs substrate was chemically etched and the remaining GaN films showed a distinct light yellow-brown coloration to the eye, and invariably showed a broad absorption tail (see inset of Fig. 1) extending from the ultraviolet into the visible spectral region which made a precise determination of the fundamental edge difficult. PL spectra recorded between 3.26 and $3.40 \mathrm{eV}$ at low intensity for different temperatures clearly showed strong near-edge features. These are shown in Fig. 2, where only the near gap spectral region is plotted because mid-gap emission reported at longer wavelengths $(\sim 550 \mathrm{~nm})$ in earlier investigations of $c$-GaN (Ref. 14) was too weak to be observed.

Two salient groups of lines appeared in the PL spectra. First, an intense, structured feature at $3.366 \mathrm{eV}$ dominated the spectrum at $6.5 \mathrm{~K}$, and broadened and shifted to lower energies as the temperature was increased. Its position shifted in a manner similar to that ascribed to the band edge in earlier research on $c$-GaN, ${ }^{14}$ decreasing by $5 \mathrm{meV}$ between 6.4 and $70 \mathrm{~K}$. In spectra recorded above $40 \mathrm{~K}$, the intensity of this peak diminished and a small thermally acti- 


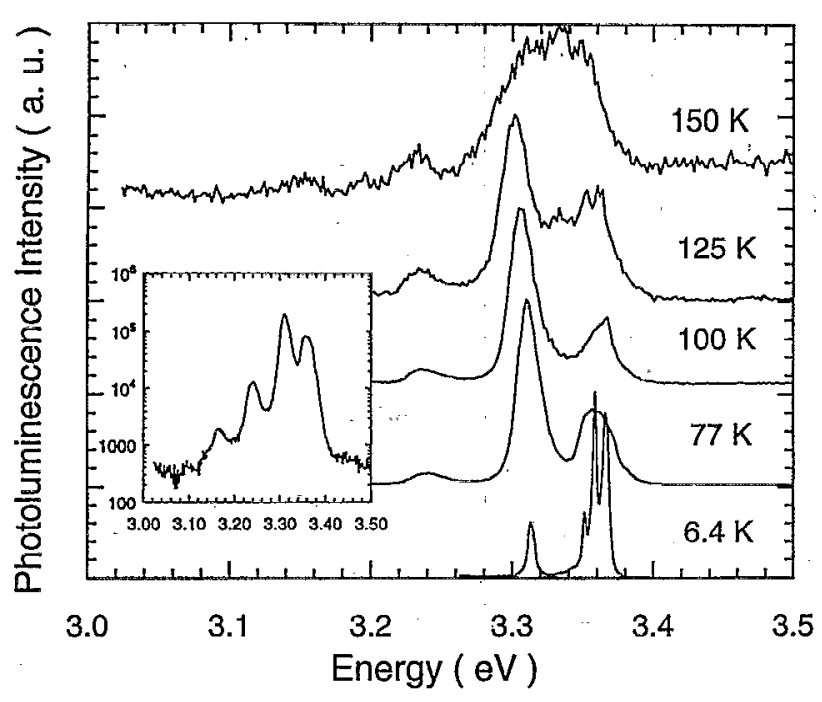

FIG. 3. Photoluminescence spectra vs temperature at high excitation intensity $\left(P=38 \mathrm{~W} / \mathrm{cm}^{2}\right.$ and $\left.l_{\mathrm{ex}}=325 \mathrm{~nm}\right)$. Inset: Logarithmic plot of the $3.31 \mathrm{eV}$ emission line and satellite intensities at $77 \mathrm{~K}$.

vated peak positioned $10 \mathrm{meV}$ higher in energy, which also tracked the band edge, became evident.

A second group of lines appeared at energies below 3.32 $\mathrm{eV}$. In this region, an intense feature accompanied by satellites was seen. To improve signal-to-noise ratio for the weaker features, spectra were recorded at elevaled excitation intensities and are shown in Fig. 3. This resulted in a considerable loss of resolution. However traces recorded this way (inset: Fig. 3) served to rather clearly establish the relationship between the electronic origin at $3.310 \mathrm{eV}$ and its three evenly spaced satellites at lower energies. At the lowest temperature, the satellites had transition energies of 3.239 , 3.164 , and $3.095 \mathrm{eV}$, and as temperature was raised the dominant shift in their positions was again in close accord with the shift in the absorption band edge. Based on the Raman splittings given above, which are very similar to those of wurtzitic $\mathrm{GaN},{ }^{18}$ the three low energy satellites can be assigned as phonon-assisted replicas of the recombination line at $3.310 \mathrm{eV}$ corresponding to the emission of one, two, and three TO phonons, respectively. (The peak at $3.366 \mathrm{eV}$ in Fig. 3 corresponds to the first group of lines described earlier, greatly broadened and saturated under these conditions.)

To provide further information on the origin of luminescence features, we recorded PL spectra for various excitation intensities at low temperature. These results are presented in Fig. 4. As excitation intensity was increased, very different behavior was observed for the two sets of lines distinguished above. First, structure on the low energy wing of the dominant spectral peak at $3.366 \mathrm{eV}$ was resolved into two additional peaks whose intensity ratio with respect to the main peak was greatly altered at high intensity. This was evidently due to a saturation effect experienced by the main peak which did not affect the subsidiary peaks, suggesting that the lines in the wing were of different origin. All three lines had

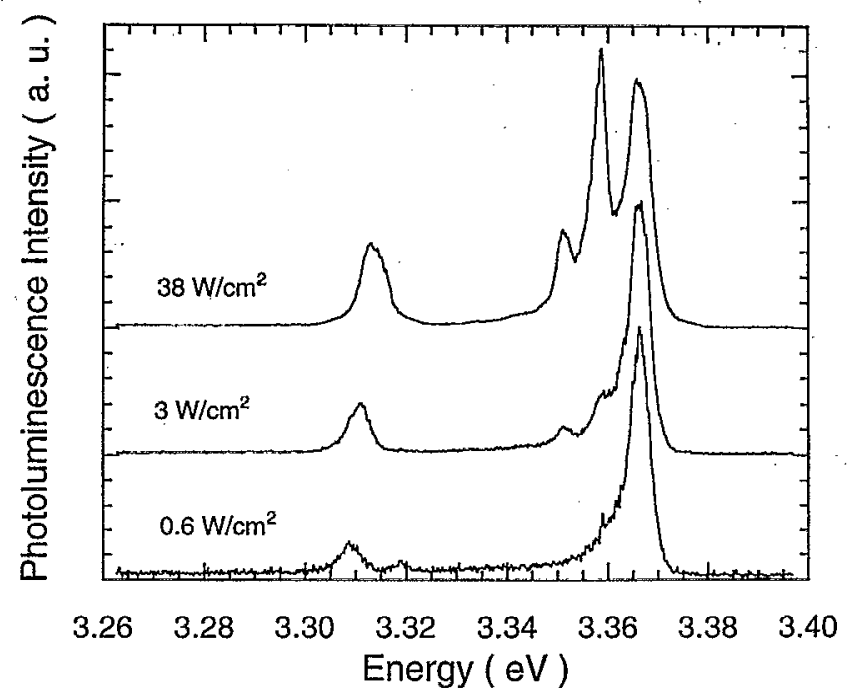

FIG. 4. Photoluminescence spectra of $\mathrm{GaN}$ vs excitation intensity, at a fixed temperature of $6.5 \mathrm{~K}\left(l_{\mathrm{ex}}=325 \mathrm{~nm}\right)$. Magnification factors for the three carves are $\times 1, \times 5, \times 40$ (top to bottom).

the common feature however of showing no shift with intensity, consistent with bound exciton emission. By contrast, the main peak at $3.310 \mathrm{eV}$ of the second group showed a significant shift to higher energy as a function of incident intensity, in a direction opposite to that of the band edge variation with temperature.

The shift of the $3.310 \mathrm{eV}$ line and its satellites to higher energy as excitation intensity is increased is an important signature of donor-acceptor pair recombination. ${ }^{20}$ This effect is explained by the saturation of distant donor-acceptor pairs with slow decay rates at high intensity and a relative increase in the proportion of near pair decays which occur both faster and at shorter wavelengths. Consequently we assign the peak at $3.310 \mathrm{eV}$ to a DA pair recombination band origin and its three satellites to phonon-assisted replicas of the pair recombination process. However we were unable to determine the donor or acceptor species responsible for this emission band.

Our interpretation of the features grouped at higher energy is as follows. The shift of the dominant line at $3.366 \mathrm{eV}$ with temperature tracks expected behavior of the band edge. ${ }^{14}$ However its temperature dependence distinguishes it from the highest energy feature at $3.376 \mathrm{eV}$ and its intensity independence is different from that of the two lower energy features in this group of lines (seen most clearly at 3.358 and $3.352 \mathrm{eV}$ in the top curve of Fig. 4). Hence we conclude there are at least three distinct types of emission evident in our PL spectra between 3.35 and $3.38 \mathrm{eV}$. The dominant line is attributed to a single bound exciton line for reasons further discussed below, although it could also arise from holes (electrons) combining with donor-(acceptor-) bound electrons (holes). The two features in the low energy wing of this line are attributed to an exciton bound to a different center. The small feature in the high energy wing of the dominant line is evidently also of excitonic origin but warrants closer 
scrutiny, since by virtue of its short wavelength it is a candidate for the free exciton emission line.

The shortest wavelength emission from our samples occurred at an energy only $10 \mathrm{meV}$ higher than the dominant PL line. Its splitting with respect to the main line is therefore quite different from either the LO or the TO phonon frequency, making it unlikely that this line is phonon-related. One potential explanation for its origin is band-to-band recombination, but the $10 \mathrm{meV}$ interval between this feature and the one next lower in energy is much smaller than the exciton binding energy, presumed to be very close to the effective mass value of $30 \mathrm{meV}$ estimated for $h-\mathrm{GaN}^{6}{ }^{6}$ Also, it could in principle result from the $\mathrm{B}$ exciton terminating on a light hole level. In $h$-GaN, the $\mathrm{G}_{7}-\mathrm{G}_{9}$ valence band splitting is only $6 \mathrm{meV}$ however, ${ }^{7}$ rendering this possibility remote. An alternative explanation would be that $10 \mathrm{meV}$ represents a donor (or acceptor) binding energy and that the emission is due to free carrier-bound carrier recombination. In this case the difference between the location of the conduction band edge and this emission feature would furnish the offset of the donor (acceptor) level from the conduction (valence) band. We note however, that recent experimental estimates of the band gap $^{21,22}$ as well as the absorption data of Fig. 1 suggest an altogether different interpretation.

The most direct determination of the gap to date ${ }^{22}$ relied on an absorption measurement and furnished a value of $E_{G}=3.30 \pm 0.02 \mathrm{eV}$ compared to $3.41 \pm 0.02 \mathrm{cV}$ for the hexagonal phase at room temperature. The difference between these two values is $D E_{G}=0.11 \mathrm{eV}$, in excellent agreement with the theoretical estimate of $0.10 \mathrm{eV}$ provided by Bloom. ${ }^{23}$ Reflectivity measurements ${ }^{21}$ are also in accord with Powell's experiment, giving a gap energy of roughly 3.35 $\mathrm{eV}$. Earlier cathodoluminescence results ${ }^{13,14}$ suggested much higher values, but were based on unconfirmed spectral analysis.

If we calculate a low temperature free exciton energy for $c$-GaN by simply subtracting the experimental $D E_{G}$ from the experimental free exciton energy in $h$-GaN, ${ }^{24}$ we obtain $E_{\text {exc }}=3.37 \pm 0.02 \mathrm{eV}$ for $c$-GaN. This corresponds very closely with our highest energy feature $(3.372 \mathrm{eV})$ at liquid helium temperature. Hence we think it most likely that the $3.372 \mathrm{eV}$ feature in our PL spectra corresponds to the free exciton line, which is thermally activated from a bound exciton or other recombination center. Evidence for thermal activation of the exciton level from the upper level of the transition at $3.366 \mathrm{eV}$ comes from plotting the intensity of this feature relative to that of the $3.366 \mathrm{eV}$ line versus temperature. When this is done using the data of Fig. 2, a fit to a Boltzmann distribution yields a value of $102 \mathrm{meV}$ for the activation energy, which corresponds precisely to the splitting between the two features. Moreover the splitting between these peaks remains constant as temperature increases, as one would expect. Hence the most consistent interpretation that we can offer for the origin of the highest energy feature is that it corresponds to the free exciton. By making use of the same value of exciton binding energy for $c-G a N$ as that estimated for $h-\mathrm{GaN}^{4}{ }^{4}$ we can infer a band gap of $E_{G}=3.376 \mathrm{eV}+0.030 \mathrm{eV}=(3.406 \pm 0.015) \mathrm{eV}$ for $c-\mathrm{GaN}$ at $6.5 \mathrm{~K}$ from our PL data.
Comparing these results with earlier cathodoluminescence spectra in MBE material, we note that the luminescence peak reported by Okumura at $325 \mathrm{~nm}$ (Ref. 15) for c-GaN/(100)GaAs does not correspond well with the features reported here, whereas the CL spectrum for $c-\mathrm{GaN} /$ (111)GaAs in the same paper may be comprised of unresolved peaks from all the spectral features identified here. Luminescence at longer wavelengths in spectra of Ref. 15 is virtually absent, just as in our MOCVD samples. Strite ${ }^{12}$ on the other hand resolved many peaks in cathodoluminescence, all quite different from ours, and reported a very strong band between 530 and $710 \mathrm{~nm}$ which they attributed to emission from midgap impurity levels. In view of the intense point defect emission observed by them in the visible spectral region, it is perhaps not surprising that defect-related emission at shorter wavelengths was also quite different from that reported here. It seems likely that the weak excitonic and DA pair spectral features identified here were either masked in the latter work by more intense emission of different origin or that electron-hole interactions were screened by ionized impurities which reduced the probability of exciton formation.

\section{CONCLUSIONS}

In summary, we have grown (200) cubic and (111) cubic/(0002) hexagonal GaN films on (100) GaAs substrates and have observed and analyzed their near band-edge optical spectra. The observed features remained invariant with material phase except for the spectrum intensity which became stronger for samples grown at higher temperatures. Excitation intensity dependence, together with the observed Raman spectrum of $\mathrm{GaN}$, have permitted us to identify a prominent DA pair recombination line at $3.310 \mathrm{eV}$ accompanied by $\mathrm{TO}$ phonon replicas. Features at $3.366,3.358$, and $3.352 \mathrm{eV}$ were attributed to distinct bound exciton lines, and from their temperature dependences the free exciton was identified and its energy deduced to be $3.376=0.003 \mathrm{eV}$ at liquid helium temperature.

\section{ACKNOWLEDGMENTS}

The authors wish to thank J. Singh for useful discussions, N. Draidia for assistance with sample preparation, and M. Yoder for his enthusiasm and encouragement of this research. Two of us (S.C.R. and S.W.B.) wish to acknowledge partial support from the National Science Foundation Science and Technology Center for Ultrafast Optical Science (STC PHY 8920108). Research on GaN growth was funded by the Office of Naval Research under contract N0014-92J1552.

\footnotetext{
${ }^{1}$ D. Pavlidis, C. H. Hong, and K. Wang, Proceedings of Workshop on Compound Semiconductors and Integrated Cckts (WOCSDICE), Cork, Ireland, May 1994, paper 1.2.

${ }^{2} \mathrm{C}$. H. Hong, K. Wang, and D. Pavlidis, Proceedings of 36th Electronic Materials Conference (EMC), Boulder, CO, June 1994, paper Q5.

${ }^{3}$ J. I. Pankove, S. Bloom, and G. Harbeke, RCA Rev. 36, 163 (1975).

${ }^{4}$ V. S. Vavilov, S. I. Makarov, M. V. Chukichev, and I. F. Chetverikova, Sov. Phys. Semicond. 13, 1259 (1979).

${ }^{5}$ H. G. Grimmeis and B. Monemar, J. Appl. Phys. 41, 4054 (1970); O. Lagerstedt and B. Monemar, J. Appl. Phys. 45, 2266 (1974).
} 
${ }^{6} \mathrm{R}$. Dingle and M. Ilegems, Solid State Commun. 9, 175 (1971).

${ }^{7}$ R. Dingle, D. D. Sell, S. E. Stokowski, and M. Ilegems, Phys. Rev. B 4, 1211 (1971).

${ }^{8}$ T. Lei, T. D. Moustakas, R. J. Graham, Y. He, and S. J. Berkowitz, J. Appl. Phys. 17, 4933 (1992).

${ }^{9}$ M. J. Paisley, Z. Sitar, J. B. Posthill, and R. F. David, J. Vac. Sci. Technol. B 7, 701 (1989).

${ }^{10}$ R. C. Powell, G. A. Tomasch, Y. W. Kim, J. A. Thorston, and J. E. Greene, Proc. MRS Symp. 162, 525 (1992).

${ }^{11}$ S. Miyoshi, K. Onabe, N. Ohkouchi, H. Yaguchi, R. Ito, S. Fukatsu, and Y. Shiraki, J. Cryst. Growth 124, 439 (1992).

${ }^{12}$ M. Mizuta, S. Fujieda, Y. Matsumoto, and T. Kawamura, Jpn. J. Appl. Phys. 25, L945 (1986).

${ }^{13}$ S. Fujieda and Y. Matsumoto, Jpn. J. Appl. Phys. 30, L1665 (1991).

${ }^{14}$ S. Strite, J. Ruan, Z. Li, A. Salvador, H. Chen, D. J. Smith, W. J. Choyke, and H. Morkoc, J. Vac. Sci. Technol. B 9, 1924 (1991).

${ }^{15}$ H. Okumura, S. Misawa, and S. Yoshida, Appl. Phys. Lett. 59, 1058 (1991).

${ }^{16} \mathrm{C}$. H. Hong, K. Wang, and D. Pavlidis, Proceedings of 21 st International
Symposium on Compound Semiconductors, San Diego, CA, Sept. 1994.

${ }^{17}$ S. Murugkar, R. Merlin, T. Lei, and T. D. Moustakas, Bull. Am. Phys. Soc. 37, 556 (1992), abstract M23-7.

${ }^{18}$ S. W. Brown, S. C. Rand, C.-H. Hong, and D. Pavlidis, Proceedings of the Materials Research Society, San Francisco, CA, April 4-8, 1994, paper D 4.26 .

${ }^{19}$ V. Lemos, C. A. Arguello, and R. C. C. Leite, Solid State Commun. 11, 1351 (1972); A. Cingolani, M. Ferrara, M. Lugara, and G. Scamarcio, Solid State Commun. 58, 823 (1986).

${ }^{20}$ D. G. Thomas, J. J. Hopfield, and W. M. Augustyniak, Phys. Rev. A 140, $202(1965)$

${ }^{21}$ H. Okumura, S. Yoshida, and T. Okahisa, Appl. Phys. Lett. 64, 2997 (1994).

${ }^{22}$ R. Powell, G. Tomasch, Y. Kim, J. Thornton, and J. Greene, Mater. Res. Soc. Symp. Proc. 162, 525 (1990).

${ }^{23}$ S. Bloom, G. Harbeke, E. Meier, and I. B. Ortenburger, Phys. Status Solidi B 66, 161 (1974).

${ }^{24}$ B. Monemar, Phys. Rev. B 10, 676 (1974). 